\title{
FOUNDATIONS OF HANKEL TRANSFORM ALGORITHMS
}

\author{
By
}

BRUCE W. SUTER

Air Force Institute of Technology, Wright-Patterson Air Force Base, Ohio

\begin{abstract}
A brief survey of existing Hankel (Fourier-Bessel) transform algorithms is presented along with a natural way to classify these algorithms. In several cases these algorithms were derived originally by methods that were unnecessarily complicated and not sufficiently general. By using operator notation and Radon transform methods, derivations and generalizations are straightforward. These improvements and generalizations are given at the appropriate places in the discussion.
\end{abstract}

1.0. Introduction. Assume that $f(r)$ satisfies the following conditions:

1) $f(r)$ is defined for $0 \leq r<\infty$.

2) $f(r)$ and $\frac{d f(r)}{d r}$ are piecewise continuous over any finite interval, except at isolated points of measure zero.

3) $f(r)$ is absolutely convergent,

$$
\int_{0}^{\infty}|f(r)| d r<\infty
$$

Definition. The $n$ th-order Hankel transform (also called Fourier-Bessel transform) of $f(r)$ satisfying 1), 2), and 3) is defined [7, 40] as

$$
\tilde{f}(u)=2 \pi \int_{0}^{\infty} r f(r) J_{n}(2 \pi r u) d r,
$$

where $J_{n}$ is a Bessel function of the first kind [45].

The integral transform (1) is expressed in operator notation by

$$
\tilde{f}(u)=\mathbf{H}_{n} f(r) \text {, }
$$

where $\mathbf{H}_{n}$ represents the $n$ th-order Hankel transform.

In a variety of applications which exhibit cylindrical symmetry, the Hankel transform of a function must be computed. These include underwater acoustics [17, 18], atomic physics [43], goephysical prospecting [13, 20], laser mode analyses [38], image processing [33], structural dynamics due to earthquakes [28], and speech processing [21]. Thus, the Hankel transform has been applied to problems in diffraction and wave propagation.

A device that will be repeatedly used throughout the paper is the following: By imposing radial symmetry on $f(x, y)$, then $f(r)$ can be described as a part of Received April 19, 1990. 
separable function $f(x, y)=f(r) \exp (\operatorname{in} \theta)$. Since the Hankel transform of $f(r)$ is desired, no generality is lost.

2.0. New classification. It is proposed that Hankel transform algorithms be systematically organized into three different categories: direct, indirect, and miscellaneous methods. Direct methods are based on the definition of the Hankel transform, while indirect methods are based on traversing Radon space while calculating the Hankel transform. Miscellaneous methods are methods that appear to have promise, but require that significant computational problems be solved before they become practical.

Direct methods, the traditional and more established ones, are a class of Hankel transform algorithms directly ascertainable from the definiton of the Hankel transform. The direct methods may be organized further into four different classes: quadrature methods, asymptotic algorithms, convolutional algorithms, and simplified two-dimensional fast Fourier transform algorithms (see Table 1).

Indirect methods refer to a class of Hankel transform algorithms based indirectly on the definition of the Hankel transform. Although this class is more recent than the direct method one, several practical algorithms have been developed. The indirect methods are organized futher into two different classes: projection-slice algorithms and filtered backprojection algorithms (see Table 1).

All of the classes of Hankel transform algorithms mentioned thus far have been utilized in at least one engineering or scientific application. Two other methods are worthy of being mentioned, although they still require more study before their usefulness can be fully determined. These miscellaneuos methods are hybrid methods and orthogonal function methods (see Table 1).

TABLE 1. Hankel transform algorithms.

\begin{tabular}{|l|l|}
\hline Category & Classes of Algorithms \\
\hline Direct & $\begin{array}{l}\text { Quadrature Methods } \\
\text { Asymptotic Algorithms } \\
\text { Convolutional Algorithms } \\
\\
\text { Simplified 2-D FFT }\end{array}$ \\
\hline Indirect & $\begin{array}{l}\text { Projection-Slice Theorem } \\
\text { Filtered Backprojection Algorithms }\end{array}$ \\
\hline Miscellaneous & $\begin{array}{l}\text { Hybrid Methods } \\
\text { Orthogonal Function Methods }\end{array}$ \\
\hline
\end{tabular}

3.0. Direct methods. In the next four subsections we cover the four classes of direct Hankel transform algorithms listed in the top third of Table 1.

3.1. Quadrature methods. Quadrature methods involve direct numerical integration [15]. An integral is approximated by a linear combination of integrand values:

$$
\int_{a}^{b} g(x) d x \approx \sum_{i=1}^{N} g\left(x_{i}\right) \omega_{i},
$$

where $x_{1}, x_{2}, \ldots, x_{N}$ are $N$ points, or abscissas, normally chosen in the interval of integration, and the numbers $\omega_{1}, \omega_{2}, \ldots, \omega_{N}$ are weights assigned to these points. 
Many times Gaussian integration is utilized to evaluate the summation over $i$, since Gaussian formulas are "best" in the sense that they integrate exactly polynomials of as high degree as possible with a formula of the form of (3).

Longman $[30,31]$ split the Hankel transform (1) into a sum of integrals of the form

$$
\tilde{f}(u)=\sum_{s=0}^{\infty} V_{s}
$$

and

$$
V_{s}=2 \pi \int_{j_{n, s-1}}^{j_{n, s}} r f(r) J_{n}(2 \pi r u) d r
$$

where $j_{n, 0}=0$ and $j_{n, s}$ is the $s$ th positive zero of the Bessel function $J_{n}(2 \pi r u)$ [45]. An approximation to (5) in a form similar to (3) is given by the summation

$$
V_{s}=2 \pi \sum_{j=1}^{N} h_{j} r_{j} f\left(r_{j}\right) J_{n}\left(2 \pi r_{j} u\right),
$$

where $r_{j}$ is the abscissa and $h_{j}$ is the weight. $V_{s}$, the terms in the summation over $s$ in Eq. (4) are often alternating in sign due to the Bessel function's oscillatory behavior, and the result may be slowly convergent. Numerous techniques exist for transforming a slowly convergent series into one more rapidly convergent. These techniques include Euler transformations, the $\varepsilon$-transformation, Aitken's $\Delta^{2}$ method, and Levin's $V$-transformation [15]. An early review of quadrature methods was provided by Cornille [14].

More recently, Chave [12] presented an iterative technique that utilized a Pade convergence acceleration step together with Patterson's [36] methods for the optimal addition of points to a quadrature formula. Chave's [12] technique permits the control of local error, defined as the difference between the result of two successive quadratures, each with a different number of abscissas. In addition, Chave [12] demonstrated that, through the utilization of the Pade approach, it is possible to numerically integrate Hankel transforms that are formally divergent. This algorithm was applied by Chave and Cox [13] to the calculation of the electromagnetic fields associated with the sea floor.

3.2. Asymptotic algorithms. Utilizing the asymptotic expansion for the Bessel function of the first kind [5],

$$
J_{n}(x) \approx[2 /(\pi x)]^{1 / 2} \cos [x-(2 n+1) \pi / 4],
$$

the Hankel transform (1) becomes

$$
\tilde{f}(u) \approx 2 \pi \int_{0}^{\infty} r f(r)[2 / \pi(2 \pi r u)]^{1 / 2} \cos [(2 \pi r u)-(2 n+1) \pi / 4] d r
$$

or equivalently

$$
\tilde{f}(u) \approx\left(2 u^{-1 / 2}\right) \int_{0}^{\infty} r^{1 / 2} f(r) \cos [2 \pi r u-(2 n+1) \pi / 4] d r .
$$


Extending this integral over $r$ from $-\infty$ to $\infty$ and utilizing the fact that $\cos \theta=$ $\operatorname{Re}[\exp (-i \theta)]$ yields,

$$
\tilde{f}(u) \approx\left(u^{-1 / 2}\right) \operatorname{Re}\left\{\exp [i(2 n+1) \pi / 4] \int_{-\infty}^{\infty}(|r|)^{1 / 2} f(|r|) \exp (-i 2 \pi r u) d r\right\} .
$$

Utilizing operator notation Eq. (7) becomes

$$
\tilde{f}(u) \approx\left(u^{-1 / 2}\right) \operatorname{Re}\left\{\exp [i(2 n+1) \pi / 4] \mathrm{F}_{1}\left[(|r|)^{1 / 2} f(|r|)\right]\right\},
$$

where $\mathbf{F}_{1}$, the (one-dimensional) Fourier transform in cartesian coordinates, is given by $[7,40]$

$$
\tilde{f}(u)=\mathbf{F}_{1} f(x)=\int_{-\infty}^{\infty} f(x) \exp (-i 2 \pi x u) d x
$$

DiNapoli and Deavenport [17] utilized Eq. (8) to approximate the $n$ th-order Hankel transform in terms of a fast Fourier transform.

Caution must be exercised when using asymptotic algorithms, since their results are only valid for large values of the output argument. When for a given application this is not a limitation, i.e., only large values of the output argument are desired, then this algorithm can be successfully applied. DiNapoli and Deavenport [17] applied this algorithm to the problem of modeling underwater acoustic propagation loss versus range.

3.3. Convolutional algorithms. Convolutional algorithms have been developed independently by Ghosh [20], Siegman [39], and Talman [43]. Let

$$
H(u)=u \tilde{f}(u),
$$

where $\tilde{f}(u)$ is the equation for the Hankel transform (1),

$$
H(u)=2 \pi \int_{0}^{\infty} r u f(r) J_{n}(2 \pi r u) d r .
$$

Assume that $f(r)=0$ for $r \leq 0$ and that $r$ and $u$ vary in the following manner,

$$
r=r_{0} \exp (-n) \text { and } u=u_{0} \exp (m) .
$$

Substituting Eq. (11) into Eq. (10) yields

$$
H(m)=2 \pi u_{0} \exp (m) \int_{-\infty}^{\infty}\left[r_{0} \exp (-n)\right]^{2} f\left[r_{0} \exp (-n)\right] J_{n}\left[2 \pi r_{0} u_{0} \exp (m-n)\right] d n .
$$

Let

$$
F(n)=\left[r_{0} \exp (-n)\right] f\left[r_{0} \exp (-n)\right]
$$

and

$$
G(m-n)=2 \pi r_{0} u_{0} \exp (m-n) J_{n}\left[2 \pi r_{0} u_{0} \exp (m-n)\right] .
$$

Then $H(m)$ becomes

$$
H(m)=\int_{-\infty}^{\infty} F(n) G(m-n) d n,
$$

since $f(r)=0$ for $r \leq 0$. Therefore,

$$
\tilde{f}(u)=H(m) / u=H(m) /\left[u_{0} \exp (m)\right]
$$


One approach to the calculation of Eq. (12) is with the Fourier convolution theorem, which states that the convolution of two functions corresponds to the product of their transforms. Hence,

$$
H(m)=\mathbf{F}_{1}^{-1}\left[\mathbf{F}_{1} F(n) \mathbf{F}_{1} G(n)\right] .
$$

Sheng and Siegman [38] utilized this approach to the problem of modeling high power lasers.

In geophysics, Hankel transforms frequently occur as a set of transforms whose integrands are related algebraically (see, for example, Verma [44]). If each of these Hankel transforms is evaluated using convolutional algorithms, then the resulting set of evaluations will be termed related convolutions [1]. In additon, after the initial direct convolution, subsequent "lagged" convolutions [2] are performed with previously computed and saved function values. Anderson [2] showed that by arranging related and lagged convolutions in a matrix, the number of function calls can be minimized. The resulting algorithm [3] is extensively utilized by geophysicists [4].

3.4. Simplified two-dimensional fast Fourier transform algorithms. The zero-order Hankel transform may be defined as the two-dimensional Fourier transform of a circularly symmetric function, and described by [7]

$$
\mathbf{H}_{0} f(\boldsymbol{r})=\mathbf{F}_{2} f(r),
$$

where $r=\left[x^{2}+y^{2}\right]^{1 / 2}$ and $\mathbf{F}_{2}$, the two-dimensional Fourier transform in cartesian coordinates, is given by $[7,40]$

$$
\tilde{f}(u, v)=\mathbf{F}_{2} f(x, y)=\int_{-\infty}^{\infty} \int_{-\infty}^{\infty} f(x, y) \exp [-i 2 \pi(x u+y v)] d x d y .
$$

Utilizing Eq. (14), Murphy and Gallagher [33] developed a simplified two-dimensional fast Fourier transform algorithm. They did not formulate the problem in a general manner, and extensions to higher orders were not apparent. Murphy and Gallagher [33] utilized this algorithm in image processing applications in which the output data are required to be in the form of a two-dimensional rectangular array for subsequent processing.

Utilizing operator notation, the framework for simplified two-dimensional fast Fourier transform algorithms will be generalized. When $f(x, y)=f(r) \exp (\operatorname{in} \theta)$, the Hankel transform is related to the two-dimensional Fourier transform of $f(r) \exp (\operatorname{in} \theta)$ :

$$
\mathbf{F}_{2} f(r) \exp (\operatorname{in} \theta)=\exp (i n \phi) i^{-n} \mathbf{H}_{n} f(r) .
$$

Proof. To see how (16) is obtained it is convenient to express the definition of the two-dimensional Fourier transform in polar coordinates,

$$
\mathbf{F}_{2} f(r, \theta)=\int_{0}^{\infty} \int_{0}^{2 \pi} f(r, \theta) \exp [-i 2 \pi r q \cos (\theta-\phi)] r d r d \theta .
$$

Let $\beta=\theta-\phi$, then

$$
\mathbf{F}_{2} f(r) \exp (i n \theta)=\exp (i n \phi) \int_{0}^{\infty} d r f(r) r \int_{0}^{2 \pi} \exp [i(n \beta-2 \pi r q \cos \beta)] d \beta .
$$


By use of the identity $J_{m}(x)=\left(i^{m} / 2 \pi\right) \int_{0}^{2 \pi} \exp [i(m \alpha-x \cos \alpha)] d \alpha$ [45], it follows that

$$
\mathbf{F}_{2} f(r) \exp (i n \theta)=\exp (i n \phi) i^{-n} 2 \pi \int_{0}^{\infty} r f(r) J_{n}(2 \pi r q) d r .
$$

This result in operator notation is

$$
\mathbf{F}_{2} f(r) \exp (\operatorname{in} \theta)=\exp (i n \phi) i^{-n} \mathbf{H}_{n} f(r) .
$$

QED

It is important to realize that if $f(r)$ is real, then $\mathbf{H}_{n} f(r)$ is a real. This suggests that a form simpler than (16) may exist. Since $\phi$ is a parameter, let $\phi$ equal zero. When $\phi$ is set to zero, no generality is lost since the functions $f(r)$ have radial symmetry. Consequently, if the Hankel transform is known for any angle $\phi$, then it is known for all $\phi$.

If $f(r)$ is real, then the Hankel transform and the two-dimensional Fourier transform are related by

$$
\mathbf{H}_{n} f(r)=\left[\exp (\operatorname{in} \phi) \mathbf{H}_{n} f(r)\right]_{\phi=0}= \begin{cases}i^{n} \mathbf{F}_{2} f(r) \cos (n \theta), & \text { if } n \text { is even } \\ i^{n} \mathbf{F}_{2} f(r) \sin (n \theta), & \text { if } n \text { is odd. }\end{cases}
$$

Proof. Since $f(r)$ is real, then $\mathbf{H}_{n} f(r)$ is real. Therefore, utilizing Eq. (16) with $\phi=0$ yields,

$$
\mathbf{H}_{n} f(r)=\operatorname{Re}\left\{i^{n} \mathbf{F}_{2} f(r) \exp (i n \theta)\right\} .
$$

This equation can be rewritten utilizing the definition of $i^{n}$.

$$
\mathbf{H}_{n} f(r)= \begin{cases}\operatorname{Re}\left\{\mathbf{F}_{2} f(r) \exp (i n \theta)\right\}, & \text { if } n \operatorname{MOD} 4=0 \\ i \operatorname{Im}\left\{\mathbf{F}_{2} f(r) \exp (i n \theta)\right\}, & \text { if } n \operatorname{MOD} 4=1 \\ -\operatorname{Re}\left\{\mathbf{F}_{2} f(r) \exp (i n \theta)\right\}, & \text { if } n \operatorname{MOD} 4=2 \\ -i \operatorname{Im}\left\{\mathbf{F}_{2} f(r) \exp (i n \theta)\right\}, & \text { if } n \operatorname{MOD} 4=3 .\end{cases}
$$

The real and imaginary parts of the Fourier transform of $f(x, y)$ are given by

$$
\operatorname{Re}\left\{\mathbf{F}_{2} f(x, y)\right\}=\mathbf{F}_{2}\left[f(x, y)+f^{*}(x, y)\right] / 2
$$

and

$$
\operatorname{Im}\left\{\mathbf{F}_{2} f(x, y)\right\}=\mathbf{F}_{2}\left[f(x, y)-f^{*}(x, y)\right] / 2 i,
$$

where $f^{*}(x, y)$ is the complex conjugate of $f(x, y)$. Then,

$$
\mathbf{H}_{n} f(r)= \begin{cases}\mathbf{F}_{2} f(r) \cos (n \theta), & \text { if } n \operatorname{MOD} 4=0 \\ i \mathbf{F}_{2} f(r) \sin (n \theta), & \text { if } n \operatorname{MOD} 4=1 \\ -\mathbf{F}_{2} f(r) \cos (n \theta), & \text { if } n \operatorname{MOD} 4=2 \\ -i \mathbf{F}_{2} f(r) \sin (n \theta), & \text { if } n \operatorname{MOD} 4=3 .\end{cases}
$$

Equivalently,

\section{QED}

$$
\mathbf{H}_{n} f(r)= \begin{cases}i^{n} \mathbf{F}_{2} f(r) \cos (n \theta), & \text { if } n \text { even } \\ i^{n} \mathbf{F}_{2} f(r) \sin (n \theta), & \text { if } n \text { odd. }\end{cases}
$$

The simplified two-dimensional fast Fourier transform algorithms are placed in perspective through the utilization of operator notation. 
4.0. Indirect methods. In the next two subsections we cover the two classes of indirect Hankel transform algorithms listed in the middle third of Table 1, and develop extensions and generalizations using Radon transform methods.

4.1. Projection-slice algorithms. Through manipulations of the two-dimensional Fourier transform, Kausel and Bouchovalas [27] arrived at the following equations:

$$
f(x)=\int_{-\infty}^{\infty} \cos (n \theta) g(r) d y
$$

and

$$
G(\xi)=i^{n} \int_{-\infty}^{\infty} f(x) \exp (-i 2 \pi \xi x) d x,
$$

where $\theta=\tan ^{-1}(y / x), r=\left(x^{2}+y^{2}\right)^{1 / 2}$, and $G(\xi)$ is an approximation to $H_{n} g(r)$.

Since Kausel and Bouchovalas [27] did not formulate the problem in a more general manner, the limitations of (18) were not apparent. It will be shown through the utilization of operator notation that Eq. (18) is only correct for Hankel transforms of even order. In spite of these errors, Kausel and Roesset [28] were able to utilize this technique to analyze the dynamics of structures due to earthquakes.

Utilizing operator notation, the framework for projection-slice algorithms will be specified in terms of the projection-slice theorem [16, 24]:

$$
\mathbf{F}_{2} f(x, y)=\mathbf{F}_{1} \mathbf{R}_{2} f(x, y),
$$

where $\mathbf{R}_{2}$, the two-dimensional Radon transform, is given by $[6,16,19,24,37]$

$$
\mathbf{R}_{2} f(x, y)=\int_{-\infty}^{\infty} \int_{-\infty}^{\infty} f(x, y) \delta(\rho-x \cos \phi-y \sin \phi) d x d y,
$$

and $\delta$ is the Dirac delta function [7, 29]. Observe that the delta function in the equation above is one-dimensional and thus the integral over the $x y$ plane reduces to an integral along the line defined by the equation

$$
\rho=x \cos \phi+y \sin \phi .
$$

By substituting Eq. (19) into (17) the connection between the Hankel transform and the projection-slice theorem becomes apparent.

$$
\mathbf{H}_{n} f(r)= \begin{cases}i^{n} \mathbf{F}_{1} \mathbf{R}_{2} f(r) \cos (n \theta), & \text { if } n \text { is even } \\ i^{n} \mathbf{F}_{1} \mathbf{R}_{2} f(r) \sin (n \theta), & \text { if } n \text { is odd. }\end{cases}
$$

It is important to note that a projection angle $\phi$ of zero degrees is assumed. Through the utilization of the definitions of the Fourier (15) and Radon (20) transforms, Eq. (21) becomes

$$
\tilde{f}(u)=i^{n} \int_{-\infty}^{\infty} \exp (-i 2 \pi \rho u) \check{f}(\rho) d \rho
$$

where

$$
\check{f}(\rho)=\left[\int_{-\infty}^{\infty} \int_{-\infty}^{\infty} f(x, y) \delta(\rho-x \cos \phi-y \sin \phi) d x d y\right]_{\phi=0}=\int_{-\infty}^{\infty} f(\rho, y) d y
$$


and

$$
\begin{aligned}
f(\rho, y) & = \begin{cases}f(r) \cos (n \theta), & \text { if } n \text { is even } \\
f(r) \sin (n \theta), & \text { if } n \text { is odd }\end{cases} \\
r & =\left[\rho^{2}+y^{2}\right]^{1 / 2},
\end{aligned}
$$

and

$$
\theta=\tan ^{-1}(y / \rho)
$$

Thus, utilizing the insight obtained from using operator notation, we see that Eq. (18) can be corrected by changing $\cos (n \theta)$ to $\sin (n \theta)$ for Hankel transforms of odd orders.

By including Tchebycheff transforms, the theoretical framework for projectionslice algorithms becomes richer. Tchebycheff transforms are related to Radon transforms by the following equation:

$$
\mathbf{R}_{2} f(r) \exp (i n \theta)=\exp (i n \phi) \mathbf{T}_{n} f(r),
$$

where $\mathbf{T}_{n}$, the $n$ th-order Tchebycheff transform, is given by

$$
\mathbf{T}_{n} f(r)=2 \int_{|\rho|}^{\infty} f(r) T_{n}(\rho / r) d r /\left[1-(\rho / r)^{2}\right]^{1 / 2},
$$

where $T_{n}$ is a Tchebycheff polynomial, Type I [5].

Proof. To see how (24) is obtained it is convenient to express the definiton of the Radon transform (20) in polar coordinates,

$$
\mathbf{R}_{2} f(r) \exp (\operatorname{in} \theta)=\int_{0}^{\infty} \int_{0}^{2 \pi} f(r) \exp (\operatorname{in} \theta) \delta[\rho-r \cos (\theta-\phi)] r d r d \theta .
$$

If the change of variable $\beta=\theta-\phi$ is made, then

$$
\mathbf{R}_{2} f(r) \exp (\text { in } \theta)=\exp (\text { in } \phi) \int_{0}^{\infty} d r f(r) r \int_{0}^{2 \pi} \exp (\operatorname{in} \beta) \delta(\rho-r \cos \beta) d \beta .
$$

The integral over $\beta$ can be evaluated using the fact that

$$
\int a(x) \delta(h(x)) d x=\sum_{i} a\left(x_{i}\right) /|d h / d x|_{x=x_{i}},
$$

where $x_{i}$ 's are zeroes of multiplicity one of $h(x)$ within the limits of integration of $x$ [26].

The zeroes of the argument of the delta function are

$$
\beta= \pm \cos ^{-1}(\rho / r), \quad r \geq \rho .
$$

By using this result the integral over $\beta$ yields

$$
\int_{0}^{2 \pi} \exp (\operatorname{in} \beta) \delta(\rho-r \cos \beta) d \beta=2 \cos \left[n \cos ^{-1}(\rho / r)\right] /\left(r\left[1-(\rho / r)^{2}\right]^{1 / 2}\right), \quad r \geq \rho .
$$

Since the Tchebycheff polynomial is related to the cosine function by $T_{n}(x)=$ $\cos \left[n \cos ^{-1}(x)\right]$, then

$$
\int_{0}^{2 \pi} \exp (\operatorname{in} \beta) \delta(\rho-r \cos \beta) d \beta=2 T_{n}(\rho / r) /\left(r\left[1-(\rho / r)^{2}\right]^{1 / 2}\right), \quad r \geq \rho .
$$


Hence,

$$
\mathbf{R}_{2} f(r) \exp (\operatorname{in} \theta)=2 \exp (\operatorname{in} \phi) \int_{|\rho|}^{\infty} f(r) T_{n}(\rho / r) d r /\left[1-(\rho / r)^{2}\right]^{1 / 2} .
$$

This result in operator notation is

$$
\mathbf{R}_{2} f(r) \exp (i n \theta)=\exp (i n \phi) \mathbf{T}_{n} f(r) .
$$

QED

Tchebycheff transforms are related to Hankel transforms by the following equation:

Proof. From (16),

$$
\mathbf{H}_{n} f(r)=i^{n} \mathbf{F}_{1} \mathbf{T}_{n} f(r) \text {. }
$$

$$
\exp (i n \phi) i^{-n} \mathbf{H}_{n} f(r)=\mathbf{F}_{2} f(r) \exp (i n \theta),
$$

and the projection-slice theorem for the two-dimensional Fourier transform (19),

$$
\exp (i n \phi) i^{-n} \mathbf{H}_{n} f(r)=\mathbf{F}_{1} \mathbf{R}_{2} f(r) \exp (\operatorname{in} \theta) .
$$

Utilizing the relationship between the Radon transform and the Tchebycheff transform (24) results in the following equation:

$$
\exp (i n \phi) i^{-n} \mathbf{H}_{n} f(r)=\exp (\operatorname{in} \phi) \mathbf{F}_{1} \mathbf{T}_{n} f(r) .
$$

Hence, this equation in operator notation is given by

$$
\mathbf{H}_{n} f(r)=i^{n} \mathbf{F}_{1} \mathbf{T}_{n} f(r) .
$$

QED

The utilization of (26) to develop projection-slice algorithms of Hankel transforms of order $n$ is presented in Suter [41, 42]. For zero order, Eq. (26) can be rewritten as

$$
\mathbf{H}_{0} f(r)=i^{n} \mathbf{F}_{1} \mathbf{A} f(r),
$$

where $\mathbf{A}$, the Abel transform, is given by

$$
\mathbf{A} f(r)=2 \int_{|\rho|}^{\infty} f(r) d r /\left[1-(\rho / r)^{2}\right]^{1 / 2}
$$

Equation (27) forms the basis for the Hankel transform algorithms of Mook [32] and Hansen [23].

Substituting the definitions of the Fourier and Techebycheff transforms in Eq. (26) yields

$$
\mathbf{H}_{n} f(r)=i^{n} \int_{-\infty}^{\infty} \check{f}(\rho) \exp (-i 2 \pi \rho u) d \rho
$$

where

$$
\check{f}(\rho)=2 \int_{|\rho|}^{\infty} f(r) T_{n}(\rho / r) d r /\left[1-(\rho / r)^{2}\right]^{1 / 2} .
$$

Assume $f(r)=0$ for $r>R$. Then, Eq. (29) can be rewritten as

$$
\check{f}(\rho)=2 \int_{|\rho|}^{R} f(r) T_{n}(\rho / r) d r /\left[1-(\rho / r)^{2}\right]^{1 / 2} .
$$


The change of variables $z^{2}=r^{2}-\rho^{2}$ yields

$$
\check{f}(\rho)=2 \int_{0}^{\left|R^{2}-\rho^{2}\right|^{1 / 2}} d z f\left[\left(z^{2}+\rho^{2}\right)^{1 / 2}\right] T_{n}\left[\rho /\left(z^{2}+\rho^{2}\right)^{1 / 2}\right] .
$$

Although Oppenheim, Frisk, and Martinez [34, 35] derived Eqs. (28) and (31), their primary interest appears to be order zero. In a companion paper to Oppenheim, Frisk, and Martinez [35], Frisk, Oppenheim, and Martinez [18] applied this algorithm to the problem of modeling ocean acoustic propagation. In support of this algorithm, Frisk, Oppenheim, and Martinez [18] developed a new measurement technique of the plane wave reflection coefficient.

4.2. Filtered backprojection algorithms. Through the use of Bessel function identities, Candel $[8,10]$ and Gopalan and Chen [22] obtained the following equations:

$$
\phi(r)=\int_{-\infty}^{\infty} \exp (i 2 \pi \xi r) f(\xi) \xi d \xi
$$

and

$$
F(r)=\int_{0}^{\pi} \phi(r \sin \theta) \exp (-\operatorname{in} \theta) d \theta
$$

where $F(r)$ is an approximation to $\mathbf{H}_{n} f(\xi)$.

Candel [8] and Gopalan and Chen [22] were concerned with zero order only, while Candel [10] was directed towards the $n$ th-order Hankel transform. Since Candel [8, $10]$ and Gopalan and Chen [22] did not formulate the problem in a more general way, the limitations of (32) and (33) were not apparent. Moreover, flaws in the derivation of the equations were compensated in the actual implementation by symmetry arguments. It will be shown that the factor $\xi$ in (32) should be $|\xi|$. In addition, a factor of $i^{-n}$ is missing in (33). In spite of these errors, Gopalan [21] successfully utilized Eqs. (32) and (33) in the area of speech processing.

The following result is a generalization of the work of Candel $[8,10]$ and Gopalan and Chen [22]. Utilizing operator notation the framework for the filtered backprojecton will be specified in terms of the relationship between the Hankel transform and the two-dimensional Fourier transform.

$$
\mathbf{F}_{2}^{-1} \tilde{f}(r, \theta)=\exp (-i n \phi) i^{n} \mathbf{H}_{n}^{-1} \tilde{f}(r),
$$

where

$$
\tilde{f}(r, \theta)=\tilde{f}(r) \exp (\operatorname{in} \theta) .
$$

This equation is obtained by taking the inverse of both sides of the equation previously given as (16). Since the Hankel transform has a Fourier kernel [40], i.e., $\mathbf{H}_{n} f(r)=\mathbf{H}_{n}^{-1} f(r)$, then Eq. (34) becomes

$$
\mathbf{F}_{2}^{-1} \tilde{f}(r, \theta)=\exp (-i n \phi) i^{n} \mathbf{H}_{n} \tilde{f}(r) .
$$

Given a function $\tilde{f}(r, \theta)$, then its inverse two-dimensional Fourier transform can be described in terms of a filtered backprojection (see for example Deans [16]). It is given by

$$
f(x, y)=\mathbf{F}_{2}^{-1} \tilde{f}(r, \theta)=\mathbf{B F}_{1}^{-1}[|r| \tilde{f}(r, \theta)],
$$


where $\mathbf{B}$, the backprojection operator, is defined by

$$
\mathbf{B} g(x \cos \theta+y \sin \theta, \theta)=\int_{0}^{\pi} g(x \cos \theta+y \sin \theta, \theta) d \theta .
$$

Let $x=k \cos \phi$ and $y=k \sin \phi$. Then Eqs. (37) and (38) become

$$
f(k, \phi)=\mathbf{F}_{2}^{-1} \tilde{f}(r, \theta)=\mathbf{B F}_{1}^{-1}[|r| \tilde{f}(r, \theta)],
$$

where

$$
\mathbf{B} g(k \cos (\phi-\theta), \theta)=\int_{0}^{\pi} g(k \cos (\phi-\theta), \theta) d \theta .
$$

Substituting Eq. (39) into (36) yields

$$
\mathbf{B F}_{1}^{-1}[|r| \tilde{f}(r, \theta)]=\exp (-i n \phi) i^{n} \mathbf{H}_{n} \tilde{f}(r) .
$$

Since $\phi$ is a parameter, let $\phi$ equal zero. When $\phi$ is set equal to zero, no generality is lost since the functions $\tilde{f}(r)$ have radial symmetry. Consequently, if the Hankel transform is known for any angle $\phi$, then it is known for all $\phi$. Hence, Eqs. (40) and (41) become

$$
\mathbf{H}_{n} \tilde{f}(r)=i^{-n} \mathbf{B F}_{1}^{-1}[|r| \tilde{f}(r, \theta)],
$$

where

$$
\mathbf{B} g(k \cos \theta, \theta)=\int_{0}^{\pi} g(k \cos \theta, \theta) d \theta .
$$

It is important to realize that the inverse Fourier transform is performed along the $r$ direction. Recall that $\tilde{f}(r, \theta)$ was assumed to have the form $\tilde{f}(r, \theta)=$ $\tilde{f}(r) \exp (\operatorname{in} \theta)$. Then utilizing the definition of the inverse Fourier transform together with Eqs. (42) and (43) yields the following pair of integral equations

$$
\mathbf{H}_{n} \tilde{f}(r)=i^{-n} \int_{0}^{\pi} h(k \cos \theta) \exp (\operatorname{in} \theta) d \theta,
$$

where

$$
h(k)=\int_{-\infty}^{\infty}|r| \tilde{f}(r) \exp (i 2 \pi r k) d r .
$$

Let $\alpha=\theta-\pi / 2$. Since the resulting integrand is an even function, the interval of integration can be halved. Hence, Eq. (44) becomes

$$
\mathbf{H}_{n} \tilde{f}(r)=2 \int_{0}^{\pi / 2} h(k \sin \alpha) \exp (\text { in } \alpha) d \alpha .
$$

Through the utilization of Bessel function identities, Higgins and Munson [25] derived Eqs. (45) and (46), which define their $n$ th-order Hankel transform algorithm.

5.0. Miscellaneous methods. In the next two subsections we cover the two classes of miscellaneous Hankel transform algorithms listed in the bottom third of Table 1 .

5.1. Hybrid methods. Hybrid methods refer to a class of Hankel transform algorithms based on the use of a slow, accurate algorithm for small values of the output variable and the use of a fast, approximate algorithm for large values of the output variable. Both the slow, accurate algorithm and the fast, approximate algorithm can be either direct or indirect method. 
Candel [9] developed a working algorithm utilizing a combination of backprojection methods and asymptotic methods together with an ad hoc technique for switching between the slow and the fast algorithms; however, the accuracy of Candel's results was data dependent. No practical hybrid method algorithms can be expected until the switching point between the slow and the fast algorithms can be chosen to maximize accuracy.

5.2. Orthogonal function methods. Orthogonal function methods refer to a class of Hankel transform algorithms based on expanding a function in terms of an orthogonal set of functions whose members have known Hankel transforms. Cavanaugh and Cook [11] developed a working algorithm using Gaussian-Laguerre polynomials. A disadvantage is that a large number of terms are required for convergence. These algorithms could be improved by selecting the orthogonal functions to minimize the number of terms required for convergence.

Summary. A new classification scheme for Hankel transform algorithms was presented. This classification is significant because: first, it specifies a way to systematically characterize Hankel transform algorithms; and, second, it provides a formalism for extending and generalizing previously stated results. Accordingly, extensions or generalizations were provided for the simplified 2-D FFT algorithms, the projectionslice algorithms, and the filtered backprojection algorithms.

\section{REFERENCES}

[1] W. L. Anderson, Numerical integration of related Hankel transforms of orders 0 and 1 by adaptive digital filtering, Geophysics 44, 1287-1305 (1979)

[2] W. L. Anderson, Fast Hankel transforms using related and lagged convolutions, ACM Trans. Math. Software 8, 344-368 (1982)

[3] W. L. Anderson, Algorithm 558: Fast Hankel transforms using related and lagged convolutions, ACM Trans. Math. Software 8, 369-370 (1982)

[4] W. L. Anderson, Computation of Green's tensor integral for three-dimensional electromagnetic problems using fast Hankel transforms, Geophysics 49, 1754-1759 (1984)

[5] G. Arfken, Mathematical Methods for Physicists, Second Edition, Academic Press, New York, 1970

[6] H. H. Barrett, The Radon transform and its applications, E. Wolf (editor), Progress in Optics, North Holland, Amsterdam, Holland, 21 219-286 (1984)

[7] R. N. Bracewell, The Fourier Transform and Its Applications, McGraw-Hill, New York, 1978

[8] S. M. Candel, An algorithm for the Fourier-Bessel transform, Comput. Phys. Comm. 23, 343-353 (1981)

[9] S. M. Candel, Dual algorithms for the fast computation of the Fourier-Bessel transform, IEEE Trans. Acoust. Speech Signal Process. 29, 963-972 (1981)

[10] S. M. Candel, Simultaneous calculation of Fourier-Bessel transforms up to order $N$, J. Comput. Phys. 44, 243-261 (1981)

[11] E. Cavanaugh and B. D. Cook, Numerical evaluation of Hankel transforms via Gaussian-Laguerre polynomial expansions, IEEE Trans. Acoust. Speech Signal Process. 27, 361-366 (1979)

[12] A. D. Chave, Numerical integration of related Hankel transforms by quadrature and continued fraction expansion, Geophysics 48, 1671-1686 (1983)

[13] A. D. Chave and C. S. Cox, Controlled electromagnetic sources for measuring electrical conductivity beneath the oceans, 1. Forward problem and model study, J. Geophysical Research 87, 5327-5338 (1982)

[14] P. Cornille, Computation of Hankel transforms, SIAM Rev. 14, 278-285 (1972)

[15] P. J. Davis and P. Rabinowitz, Methods of Numerical Integration, Second Edition, Academic Press, New York, 1984 
[16] S. R. Deans, The Radon Transform and Some of Its Applications, Wiley, New York, 1983

[17] F. R. DiNapoli and R. L. Deavenport, Theoretical and numerical Green's function field solution in a plane multilayered medium, J. Acoust. Soc. Amer. 67, 92-105 (1980)

[18] G. V. Frisk, A. V. Oppenheim, and D. R. Martinez, A technique for measuring the plane wave reflection coefficient of the ocean bottom, J. Acoust. Soc. Amer. 68, 602-612 (1980)

[19] I. M. Gel' fand, M. I. Graev, and N. Ya. Vilenkin, Generalized Functions, Vol. 5, Academic Press, New York, 1966

[20] D. P. Ghosh, The application of linear filter theory to the direct interpretation of geoelectrical resistivity sounding measurements, Geophysical Prospecting 19, 192-217 (1971)

[21] K. Gopalan, Fournier-Bessel expansion: Numerical evaluation and application in the representation and feature extraction of speech signals, Ph. D. Dissertation, University of Akron, Akron, Oh., 1984

[22] K. Gopalan and C. S. Chen, Fast computation of zero order Hankel transform, J. Franklin Inst. 316, 317-326 (1983)

[23] E. W. Hansen, Fast Hankel transform algorithm, IEEE Trans. Acoust. Speech Signal Process. 33, 666-671 (1985), Errata 34, 623-624 (1986)

[24] S. Helgason, The Radon Transform, Birkhauser, Boston, Mass., 1980

[25] W. E. Higgins and D. C. Munson, An algorithm for computing general integer order Hankel transforms, IEEE Trans. Acoust. Speech Signal Process. 35, 86-97 (1987)

[26] J. D. Jackson, Mathematics for Quantum Mechanics, W. A. Benjamin, New York, 1962

[27] E. Kausel and G. Bouchovalas, Computation of Hankel transforms using the fast Fourier transform algorithm, Massachusetts Institute of Technology, Civil Engineering Department, Research Report R79-12, 1979

[28] E. Kausel and J. M. Roesset, Stiffness matrices for layered soils, Bull. Seismol. Soc. Amer. 71, 1743-1761 (1981)

[29] M. J. Lighthill, Introduction to Fourier Analysis and Generalized Functions, Cambridge University Press, Cambridge, England, 1962

[30] I. M. Longman, Note on a method for computing infinite integrals of oscillatory functions, Proc. Cambridge Philos. Soc. 52, 764-768 (1956)

[31] I. M. Longman, Tables for the rapid and accurate numerical evaluation of certain infinite integrals involving Bessel functions, Mathematical Tables and Other Aides to Computation 11, 166-180 (1957)

[32] D. R. Mook, An algorithm for the numerical evaluation of the Hankel and Abel transforms, IEEE Trans. Acoust. Speech Signal Process. 31, 979-985 (1983)

[33] P. K. Murphy and N. C. Gallagher, Fast algorithm for the computation of the zero-order Hankel transform, J. Opt. Soc. Amer. 73, 1130-1137 (1983)

[34] A. V. Oppenheim, G. V. Frisk, and D. R. Martinez, An algorithm for the numerical evaluation of the Hankel transform, Proc. IEEE 66, 264-265 (1978)

[35] A. V. Oppenheim, G. V. Frisk, and D. R. Martinez, Computation of the Hankel transform using projections, J. Acoust. Soc. Amer. 68, 523-529 (1980)

[36] T. N. L. Patterson, The optimum addition of points to quadrature formulae, Math. Comp. 22, 847-856 (1968), Errata 23, 892 (1969)

[37] J. Radon, Uber die Bestimmung von Funktionen durch ihre Integralwerte langs gewisser Mannigfaltigkeiten, Brie Sachsische Akademie der Wissenschaften, Leipzig, Math-Phys. K1 69, 262-267 (1917)

[38] S. Sheng and A. E. Siegman, Nonlinear-optical calculations using fast-transform methods: Second harmonic generation with depletion and diffracton, Phys. Rev. 21, 599-606

[39] A. E. Siegman, Quasi fast Hankel transform, Optics Letters 1, 13-15 (1977)

[40] I. N. Sneddon, The Use of Integral Transforms, McGraw-Hill, New York, 1972

[41] B. W. Suter, Some Numerical Applications of the Radon Transform, Ph. D. Dissertation, University of South Florida, Tampa, Fl., 1988

[42] B. W. Suter, Fast $n$ th-order Hankel transform algorithm, IEEE Trans. Signal Process. 39, 532-536 (1991)

[43] J. D. Talman, Numerical Fourier and Bessel transforms in logarithmic variables, J. Comput. Phys. 29, 35-48 (1978)

[44] R. K. Verma, Detectability by electromagnetic sounding systems, IEEE Trans. on Geoscience Electronics 15, 232-251 (1977)

[45] G. N. Watson, A Treatise on the Theory of Bessel Functions, Second edition, Cambridge University Press, Cambridge, England, 1966 